Y. Cazals

\title{
Effects of Ginkgo biloba extract (EGb 761) on cochlear vasculature in the guinea pig: morphometric measurements and laser Doppler flowmetry
}

Received: 9 February 1995 / Accepted: 26 April 1995

\begin{abstract}
Ginkgo biloba extract (EGb 761) was administered orally for 4 or 6 weeks to healthy adult guinea pigs. Animals were then decapitated under deep ketamine anesthesia. Post-mortem morphometric measurements of cochlear vessels in the spiral lamina revealed a vasodilating effect of the extract in four of ten animals following 6 weeks of treatment. In vivo testing of the effect of 4 or 6 weeks of treatment with EGb 761 was monitored with laser Doppler flowmetry of the cochlear blood flow under pathological conditions. Results demonstrated that EGb 761 partly counteracted sodium salicylate-induced decreases in cochlear blood flow (CBF) and enhanced CBF increases induced by hypoxia. These findings indicate that EGb 761 may help to improve oxygenation in cochleas with compromised blood flow.
\end{abstract}

Key words Cochlear blood flow $\cdot$ Hypoxia Ototoxicity · Laser Doppler flowmetry · Ginkgo biloba extract

\section{Introduction}

Cochlear microcirculation involves a complex vascular network [2] to maintain inner ear homeostasis and, more specifically, ion and oxygen levels in the cochlear fluids. Experimental evidence has shown that auditory function is highly dependent on normal cochlear metabolism [24, 26,

\section{A. Didier}

Department of Otolaryngology, Kresge Hearing Research Institute, University of Michigan, Ann Arbor, MI 48109-0506, USA

M.-T. Droy-Lefaix

Ipsen Institute, F-75781 Paris cedex 16, France

C. Aurousseau $\cdot$ Y. Cazals

Laboratoire d'Audiologie Expérimentale, Inserm U 229,

Hôpital Pellegrin, F-33076 Bordeaux, France

A. Didier (ब)

Laboratoire de Physiologie Neurosensorielle,

Université Claude Bernard, 43 bld du 11 Novembre 1918,

F-69622 Villeurbanne, France
28]. Many inner ear disorders, including noise-induced hearing loss $[12,33,40]$, some forms of sudden deafness [15] and presbycusis [14], are thought to be at least partly due to an impairment of microcirculation. Such therapeutic strategies as use of dilating drugs, hemodilution and $\mathrm{CO}_{2}$ inhalation [13] have attempted to improve cochlear blood flow $(\mathrm{CBF})$, and thus cochlear oxygenation.

With the development of laser Doppler (LD) flowmetry [25, 27], dynamic measurements of CBF have yielded basic data on CBF regulation $[5,21,24,29,34]$ and have provided an experimental basis for evaluating the effectiveness of clinical treatment $[17,20,22,30,31]$. Further improvements to this experimental approach include identification and development of animal models of vascular pathologies of the cochlea $[8,18,33]$.

EGb 761 is a free radical scavenger which is prepared from green leaves of Ginkgo biloba trees and contains such flavenoids substances as ginkgo-flavone glucosides and ginkgolide terpenoids [9]. Experiments in the present study were conducted to examine the effect of EGb 761 (Ipsen Laboratories, Paris, France) on the vasculature of normal guinea pig cochleas and cochleas with experimentally impaired CBF.

A first set of morphometric measurements investigated post-mortem vessel diameters in the stria vascularis and spiral lamina following long-term treatment with EGb 761. The positive results of this study led us to set up complementary experiments using LD dynamic measurements of CBF in two different experimental models of vascular disturbances: sodium salicylate-induced decreases in CBF and hypoxia-induced increases in CBF.

\section{Materials and methods}

Experimental protocols were reviewed and approved by the University of Michigan's Committee on the use and care of animals.

\section{Morphometry}

Seventeen healty adult pigmented guinea pigs, weighing 250-350 g, were used. Group $1(n=10)$ received oral EGb 761 daily, at a dose 
Table 1 Laser Doppler flowmetry: experimental groups and treatments given (+ treatment, - no treatment.)

\begin{tabular}{llll}
\hline & $\begin{array}{l}\text { EGb 761 } \\
(100 \mathrm{mg} / \mathrm{kg})\end{array}$ & $\begin{array}{l}5 \text { min hypoxia } \\
\left(12 \% \mathrm{O}_{2} \text { in N }\right)\end{array}$ & $\begin{array}{l}\text { Sodium salicylate } \\
(200 \mathrm{mg} / \mathrm{kg})\end{array}$ \\
\hline Group 1 & + & + & + \\
& $(4$ weeks $)$ & $(n=3)$ & $(n=4)$ \\
Group 2 & + & + & + \\
& $(6$ weeks $)$ & $(n=4)$ & $(n=4)$ \\
Group 3 & - & + & + \\
& & $(n=3)$ & $(n=6)$ \\
Group 4 & - & - & - \\
& & & $(n=4)$
\end{tabular}

of $100 \mathrm{mg} / \mathrm{kg}$ for 6 weeks. Controls (group 2, $n=7$ ) received water instead of EGb 761 .

At the end of treatment, the animals of both groups were killed by decapitation under deep ketamine anesthesia $(150 \mathrm{mg} / \mathrm{kg})$. The auditory bullae were excised and quickly fixed with osmium tetroxide in cacodylate buffer. The stria vascularis (excluding the spiral ligament and prominence) and the osseous spiral lamina were dissected out from the first and third turns of the cochlea and prepared for light microscopy. The areas of the vessels were measured in 4-8 microscopic fields for each sample, using an image analyser (Biocom, Les Ulis, France). Results were expressed as vascular density given by the ratio of the area of the vessels to the area of the measured field [4].

\section{CBF LD flowmetry}

Twenty-one pigmented guinea pigs, weighing 250-350 g were used (Table 1). Group $1(n=4)$ was treated for 4 weeks with oral $\mathrm{EGb} 761$ at a dose of $100 \mathrm{mg} / \mathrm{kg}$. Group $2(n=4)$ received the same treatment for 6 weeks. At the end of the treatment, animals in groups 1 and 2 were anesthetized, prepared for LD flowmetry (see below) and injected intramuscularly with freshly dissolved sodium salicylate in saline at a dose of $200 \mathrm{mg} / \mathrm{kg}$. Two control groups were used: group $3(n=6)$ received sodium salicylate but no $\mathrm{EGb}$
761. Group $4(n=4)$ was given saline instead of sodium salicylate and no EGb 761 .

The same animals were used to test the effect of EGb 761 on the CBF response to hypoxia. Animals from group $1(n=3)$, group $2(n=4)$ and half of group $3(n=3)$ were subjected to 5 min hypoxia induced by breathing a gas mixture containing $12 \%$ oxygen in nitrogen prior to administration of sodium salicylate. Three animals from group 3 were also used to check that the hypoxic episode did not change the subsequent response to sodium salicylate. Animals were allowed to recover for at least $10 \mathrm{~min}$ following the end of the hypoxic period before being injected with sodium salicylate.

After treatment with EGb 761 and before exposure to hypoxia and/or treatment with sodium salicylate animals were anesthetized with intraperitoneal sodium pentobarbital $(7.5 \mathrm{mg} / \mathrm{kg})$ and intramuscular fentanyl and droperidol (Innovar-vet, Pitman-Moore, Mundelien, Ill., $0.2 \mathrm{ml} / \mathrm{kg}$ ). One auditory bulla was surgically exposed and an LD probe (TSI, Vasamedics, St Paul, Minn.) placed over the bony wall of the first cochlear turn. Animals were tracheotomized and breathed freely room air or the hypoxic gas mixture delivered through a plastic tube loosely surrounding the tracheal tube. Body temperature was maintained at $38^{\circ} \mathrm{C}$ with a thermoregulated heating pad.

$\mathrm{CBF}$ and systemic blood pressure (BP) were monitored continuously and recorded on a chart recorder. The computed ratio of $\mathrm{CBF}$ to $\mathrm{BP}$ as cochlear whole organ vascular conductance (VC) was also recorded continuously. VC was used to reflect $\mathrm{CBF}$ changes not directly related to systemic BP changes. All experimental manipulations were performed after recording stable baseline values for $\mathrm{CBF}$ and $\mathrm{BP}$ over a 10 -min period. $\mathrm{BP}$ and $\mathrm{CBF}$ were recorded over $90 \mathrm{~min}$ following the administration of sodium salicylate.

\section{Results}

\section{Morphometry}

No difference was found in the vascular density of cochlear vessels in the stria vascularis between treated and control guinea pigs (Fig. 1A).

In the spiral lamina (Fig. 1B) no statistical difference in vascular density was demonstrable when comparing the
Fig. 1A, B Vascular density over the first and third turns of the cochlea in guinea pigs treated with EGb 761 for 6 weeks prior to sacrifice. The area in the box indicates the lower and upper limits of control values. A Vessels of the stria vascularis. B Vessels of the spiral lamina. Symbols represent individual animals

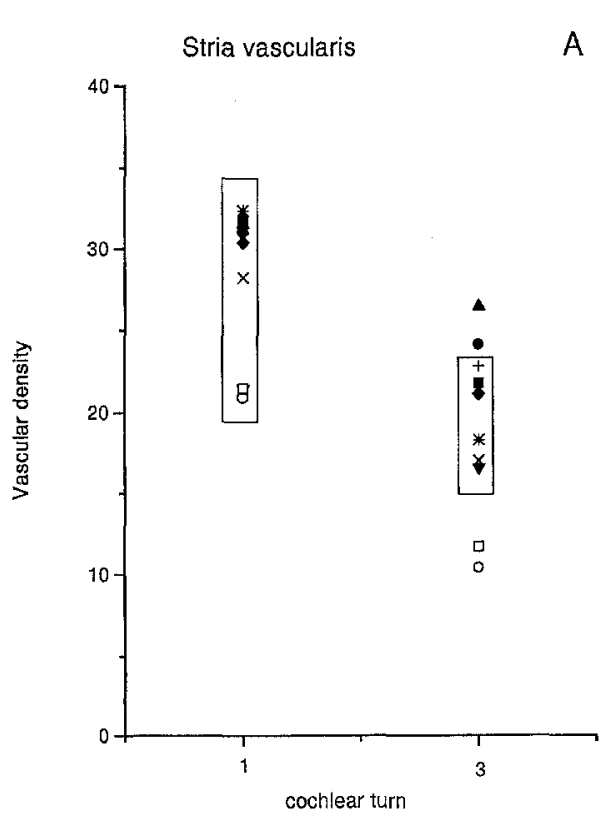

A

Spiral Lamina

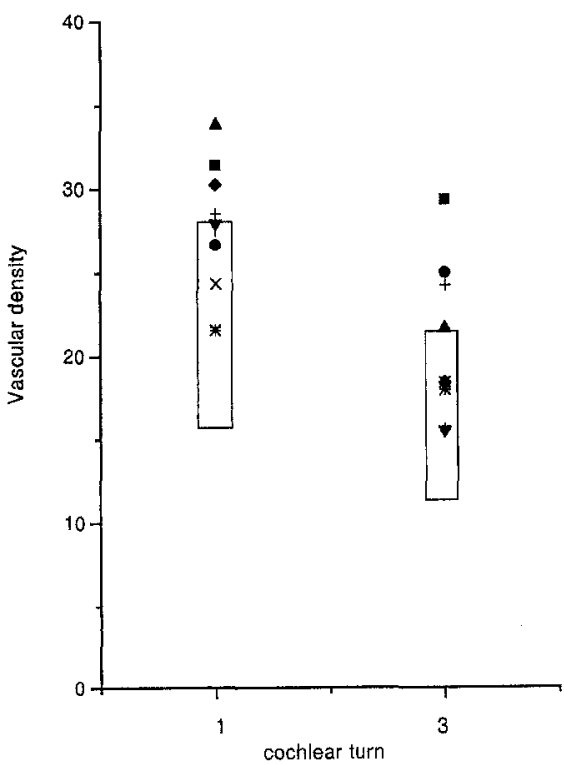


Table 2 Blood pressure expressed as percent of baseline value (SD) over a 90 -min observation period following sodium salicylate $(200 \mathrm{mg} / \mathrm{kg})$ or saline administration

\begin{tabular}{|c|c|c|c|c|c|c|c|}
\hline & \multicolumn{7}{|c|}{ Time post-injection (min) } \\
\hline & 5 & 15 & & 30 & 60 & 90 & \\
\hline $\begin{array}{l}\text { EGb } 7614 \text { weeks+sodium } \\
\text { salicylate }(n=4)\end{array}$ & $97(3.69)$ & 97 & $(7.67)$ & $94(8.66)$ & $94(2.87)$ & 85 & $(6.39)$ \\
\hline $\begin{array}{l}\text { Group } 2 \\
\text { EGb } 7616 \text { weeks+sodium } \\
\text { salicylate }(n=4)\end{array}$ & $102(2.21)$ & 109 & $(8.53)$ & $104(8.65)$ & $105(8.98)$ & 97. & $(13.32)$ \\
\hline $\begin{array}{l}\text { Group } 3 \\
\text { Sodium salicylate }(n=6) \\
\text { Group } 4 \\
\text { Control (saline, } n=4 \text { ) }\end{array}$ & $104(7.6)$ & 107 & $(5.85)$ & $\begin{array}{l}103(3.93) \\
92(7.63)\end{array}$ & $\begin{array}{l}99(4.35) \\
91(5.59)\end{array}$ & 91 & $(1.87)$ \\
\hline
\end{tabular}

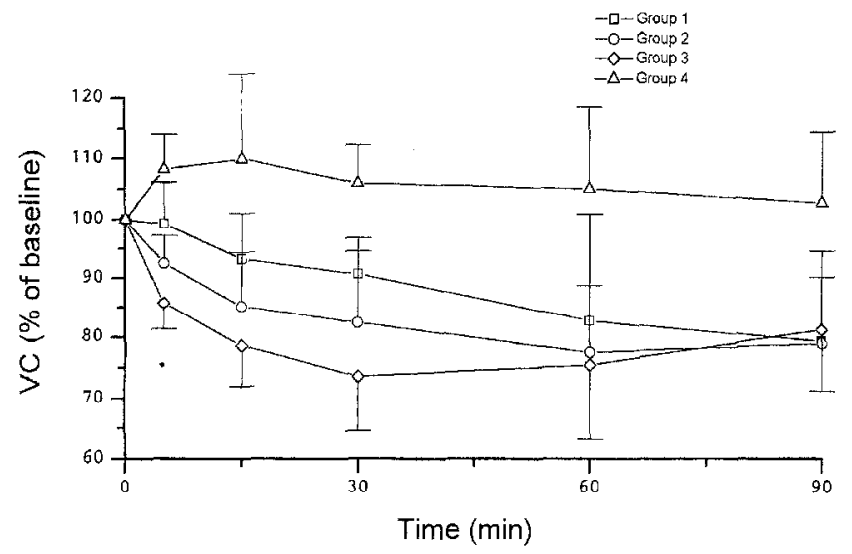

Fig. 2 Variations of vascular conductance $(V C) \pm$ SD across time following administration of sodium salicylate $(200 \mathrm{mg} / \mathrm{kg}$ i.m.) in animals treated with $\mathrm{EGb} 761$ for 4 weeks (square, group 1), 6 weeks (circle, group 2), untreated animals (diamond, group 3), and animals receiving saline instead of salicylate (triangle, group 4)

mean diameter in group 2 animals versus group 1 . However, four of ten animals in group 1 showed a higher vascular density in the first turn than the highest control value. The same was true for the third turn in four cases. Nonetheless, the other treated animals in this group showed vessel diameters within the upper range of the control values.

\section{$\mathrm{CBF}$ responses to sodium salicylate}

Percent changes from baseline of CBF were affected by $\mathrm{BP}$ changes (Table 2). These were small over the observation period (about 10\%) and consisted mostly of a decrease over time that was of a similar magnitude in the different experimental groups. $\mathrm{VC}$ (i.e. the ratio $\mathrm{CBF} / \mathrm{BP}$ ) therefore mostly reflected CBF changes normalized by the small changes in BP inherent to the experimental preparation.

Results are summarized in Fig. 2. In the control group (group 4) that received only saline, no significant variation in measured parameters was observed post-injection. In group 3, which received sodium salicylate alone, a decrease in CBF was observed, as previously described [8]. $\mathrm{VC}$ decreased over the first 30 min following administration of the drug and then stabilized at about $75 \%$ of its

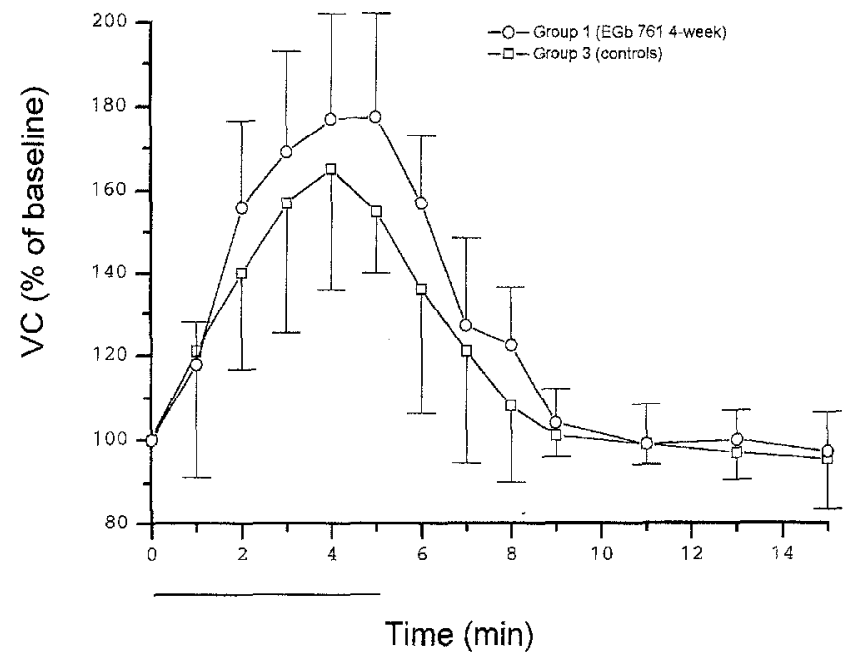

Fig. 3 Variations of VC $\pm \mathrm{SD}$ across time in response to $5 \mathrm{~min}$ of hypoxia (mark along the abscissa). Animals treated with EGb 761 for 4 weeks (circle, group 1) versus untreated animals (square, group 3)

pre-treatment level. No recovery could be observed over the $90 \mathrm{~min}$ of the observation period. In groups 1 and 2 (EGb 761-treated animals), the decrease in VC was slower than in group 3. Statistical significance using analysis of variance (ANOVA, $P<0.05$ ) was reached in group 2 at 5 , 15 and 30 min following administration of sodium salicylate when compared with findings in group 3 .

\section{CBF responses to hypoxia}

In response to hypoxia, control animals (group 3) showed a systematic increase in CBF that was as much as $130 \%$ of baseline values despite a significant decrease in BP (of about $20 \%$ ). As a result, VC reached values of about $60 \%$ above baseline (Fig. 3). In treated animals (group 1), the increase in $\mathrm{VC}$ induced during the whole hypoxic period was slightly higher than the increase found in the controls. Findings in group 2 (6 weeks of EGb 761 treatment) were essentially similar to those observed in guinea pigs treated for 4 weeks (not shown). 


\section{Discussion}

Sodium salicylate induces a reversible hearing loss in humans, when used at medium to high doses. Its mechanism of toxicity has been partly clarified recently in animal models, showing that it causes a decrease in the LD-measured CBF. Restoration of $\mathrm{CBF}$ with hydralazine as vasodilator during sodium salicylate intoxication reduces salicylate-induced hearing loss, indicating that decreased CBF contributes to the hearing loss $[8,16]$. In addition, electrophysiological and anatomical studies at an ultrastructural level point to the hair cells as a target of salicylate toxicity through an alteration of outer hair cell ionic conductance $[32,36,39]$. For this reason we examined CBF responses to acute administrations of sodium salicylate in guinea pigs chronically treated with EGb 761 as compared with untreated animals.

Low oxygenation of the cochlea is also known to alter hearing $[7,11,28]$. Such a condition can be viewed as one of the most severe forms of vascular impairment in the cochlea. A number of studies have indirectly associated hypoxia with noise-induced hearing loss. Thorne and Nuttall [41] showed decreased endolymphatic oxygenation and LD-measured CBF. Another study by Shone's group [37] on the aging ear showed that noise-induced temporary and permanent threshold shifts could be eliminated by oxygen therapy before or during noise exposure. We thus produced acute short-term hypoxia and investigated the effect of EGb 761 on CBF responses.

\section{EGb 761-induced effects on vascular density}

Morphometric data showed that EGb 761 induced vasodilation of the vessels of the spiral lamina in some cases that was not found for vessels of the stria vascularis. This differential effect of the extract on the two networks of cochlear vessels could possibly be related to the sympathetic innervation present. Indeed, anatomical investigations have revealed that the sympathetic fibers innervate spiral lamina vessels, radiating arterioles and venules but do not pass beyond the interscala septae $[3,38]$. The lateral wall vessels also do not seem to be associated with the sympathetic innervation. Consistent with this, pharmacological experiments have shown that a sympatholytic drug can increase vessel diameters in the spiral lamina but not in the stria vascularis [6].

The influence of the sympathetic innervation on CBF is most probably vasoconstrictive, as found in pharmacological [29] and physiological studies [21, 34]. EGb 761 likely exerts an antagonistic action on the cochlear sympathetic nerve to the spiral lamina vessels. This is supported by the finding that EGb 761 decreased norepinephrine-induced contraction in preparations of rabbit saphenous arteries with intact endothelium [1]. In addition, EGb 761 given intraveneously suppressed arteriolar spasm in the brain following topical application of autologous serum with normalization of the vessel diameters [35].
EGb 761-induced effects on CBF response to sodium salicylate

Sodium salicylate is believed to decrease CBF through its known inhibitory action on prostaglandin (PG) synthesis [42] occurring in the cochlea [10] and through increased levels of leukotrienes as arachidonic acid metabolites of the lipooxygenase pathway [16]. Our results show that the time course of sodium salicylate-induced decreases in CBF was slowed by EGb 761. Its dilating effect on the cochlear vessels through interaction with the sympathetic system was considered to partly counteract the action of sodium salicylate. This is supported by the potentiation of sodium salicylate ototoxicity by sympathomimetic agents [6]. In addition, a potentiation by EGb 761 of endothelium-derived relaxing factor (EDRF) release from endothelium has been shown on rabbit arteries [1].

Arachidonic acid metabolism through the lipooxygenase pathway probably generates free radicals [19]. Since increased levels of leukotrienes have been reported in the cochlea following salicylate administration [16], an additional mechanism of action of EGb 761 under salicylate intoxication may involve its free radical scavenger properties providing protection to endothelial cells from damage induced by free radicals. A positive action of $E G b$ 761 on hearing under salicylate intoxication can also be inferred from a previous report [9] showing that increasing CBF by hydralazine as a dilating agent reduces salicylate-induced hearing loss.

In our animals treated with EGb 761 for 6 weeks, the time course of the decrease in CBF in response to sodium salicylate was delayed, but this occurred to a lesser extent than in the guinea pigs treated for 4 weeks. Considering the variability of the response to sodium salicylate, further studies require the use of larger groups of animals to prove statistical significance.

EGb 761-induced effects on CBF responses to hypoxia

The increase in CBF induced in our animals by breathing a low oxygen mixture is very similar to the effect on CBF produced by carbon monoxide ventilation in the guinea pig [11]. This demonstrates the ability of CBF to be regulated locally, independent of systemic BP changes and even counteracting these by autoregulation. Oxygen measurements in the endolymph also suggest that oxygen levels are subject to some autoregulation [23]. The increased CBF during hypoxia probably represents a compensatory mechanism aimed at maintaining oxygen levels as high as possible. The CBF response to hypoxia then constitutes an experimental manipulation during which the responsiveness of CBF can be assessed with its local mechanisms of regulation. In our study, EGb 761 seemed to enhance this autoregulatory response while leaving the systemic BP response unchanged. This suggests that EGb 761 can exert a local action on CBF even when given systemically. This is of importance since many other dilating drugs given systemically slightly increase CBF but in actuality create a systemic hypotension [29]. 
Fechter et al. [11] reported that levels of carboxyhemoglobin and related hearing loss increased further than CBF with increasing levels of hypoxia. This led to the suggestion of an upper limit to the compensatory response. When comparing the value reported by Fechter et al. with our unpublished data using higher levels of hypoxia, the magnitude of the increase in $\mathrm{CBF}$ in our study suggests that the compensatory response found might be close to its maximum and is brought up to this maximum by EGb 761 treatment. Optimal effectiveness of EGb 761 would also occur for milder hypoxic levels especially since these are probably encountered more often in clinical practice.

Acknowledgements The laser Doppler flowmetry was performed in the Microcirculation Laboratory at the Kresge Hearing Research Institute (Director, J. Miller), University of Michigan. The microcirculation laboratory is directed by Dr. A.L. Nuttall. The authors would like to thank Dr. Nuttall for this and his helpful review of the manuscript. This research was supported by funds from NIH research grant R01-DC00105 and grants from Ipsen Institute, Paris, France.

\section{References}

1. Auguet M, Delaflotte S, Chabrier P-E, Braquet P (1994) EGb 761 and the regulation of the vascular tone. In: Clostre, DeFeudis (eds) Advances in Ginkgo Biloba extract research, vol 3. Cardiovascular effects of Ginkgo biloba extract (EGb 761). Elsevier, Paris, pp 19-26

2. Axelsson A (1971) The cochlear blood vessels in the guineapig at different ages. Acta Otolaryngol (Stockh) 72:172-181

3. Brechtelsbauer PB, Prazma J, Garret CG, Carrasco VN, Pillsbury HC (1990) Catecholaminergic innervation of the inner ear. Otolaryngol Head Neck Surg 103:566-574

4. Carlisle Shaddok L, Hamernik RP, Wright CG (1985) A morphometric technique for analysis of cochlear vessels. Hear Res 20:109-117

5. Carrasco VN, Prazma J, Faber JE, Trana RJ, Pillsbury HC (1990) Cochlear microcirculation. Effect of adrenergic agonists on arteriole diameter. Arch Otolaryngol Head Neck Surg 116: $411-417$

6. Cazals Y, Li XQ, Aurousseau C, Didier A (1988) Acute effects of noradrenaline related agents on the ototoxicity of aspirin: an experimental study in the guinea-pig. Hear Res 36:89-96

7. Cazals Y, Wu ZY, Horner K (1994) Alterations of auditory nerve responses by hypoxia in normal and hydropic ears of awake guinea-pigs. Hear Res 77:177-182

8. DeFeudis FV (1991) Ginkgo biloba extract (EGb 761): pharmacological activities and clinical applications. Elsevier, Paris

9. Didier A, Nuttall AL, Miller JM (1993) The vascular component of sodium salicylate ototoxicity in the guinea pig. Hear Res 69:199-206

10. Escoubet B, Amsallem P, Ferrary E, Tran Ba Huy P (1985) Prostaglandin synthesis by the cochlea of the guinea-pig. Influence of aspirin, gentamicin and acoustic stimulation. Prostaglandins 29:589-599

11. Fechter DL, Thorne PR, Nuttall AL (1987) Effects of carbon monoxide on cochlear electrophysiology and blood flow. Hear Res 27:37-45

12. Hawkins JE (1971) The role of vasoconstriction in noise-induced hearing loss. Ann Otol Laryngol 80:903-913

13. Hultcrantz E (1988) Clinical treatment of vascular inner ear diseases. Am J Otolaryngol 9:317-322

14. Johnson A, Hawkins JE (1972) Vascular changes in the human inner ear associated with aging. Ann Otol Rhinol Laryngol $81: 364-376$
15. Johnson A, Hawke M, Berger G (1984) Sudden deafness, vertigo due to inner ear hemorrhage: a temporal bone case report. J Otolaryngol 13:201-207

16. Jung TTK, Miller SK, Rozehnal S, Woo HY, Park YM, Baer W (1992) Effect of round window membrane application of salicylate and indomethacin on hearing, levels of arachidonic acid metabolites in perilymph. Acta Otolaryngol (Stockh) 493: 81-87

17. Kallinen J, Didier A, Miller JM, Nuttall AL, Grénman R (1991) The effect of $\mathrm{CO}_{2^{-}}, \mathrm{O}_{2}$-gas mixtures on laser Doppler measured cochlear and skin blood flow in guinea-pigs. Hear Res 55:255262

18. Kimura RS (1988) Animal models of inner ear vascular disturbancies. Am J Otolaryngol 7:130-139

19. Kuzuya T, Hoshida S, Kim Y, Oe H, Hori M, Kamada T, Tada M (1993) Free radical generation coupled with arachidonate lipoxygenase reaction relates to reoxygenation induced myocardial cell injury. Cardiovase Res 27:1056-1060

20. Laurikainen EA, Nuttall AL, Miller JM, Quirk WS, Virolainen E (1992) Experimental basis for lidocaine therapy in cochlear disorders. Acta Otolaryngol (Stockh) 112:800-809

21. Laurikainen EA, Kim D, Didier A, Ren T, Miller JM, Quirk WS, Nuttall AL (1993) Stellate ganglion drives sympathetic regulation of cochlear blood flow. Hear Res 64:199-204

22. Laurikainen EA, Miller JM, Quirk WS, Kallinen J, Ren T, Nuttall AL, Grenman R, Virolainen E (1993) Betahistine-induced vascular effects in the rat cochlea. Am J Otol 1993 14:24-30

23. Lawrence M, Nuttall AL, Burgio PA (1977) Oxygen reserve, autoregulation in the cochlea. Acta Otolaryngol (Stochk) 83: $146-152$

24. Miller JM, Dengerink H (1988) Control of inner-ear blood flow. Am J Otolaryngol 9:302-316

25. Miller JM, Marks NJ, Goodwin PC (1983) Laser Doppler measurements of cochlear blood flow. Hear Res 11:385-394

26. Misrahi GA, Shinabarger EW, Arnold JE (1958) Changes in cochlear endolymphatic oxygen availability, action potential and microphonics during, following asphyxia, hypoxia, exposure to loud sounds. J Acoust Soc Am 30:701-704

27. Nuttall AL (1988) Cochlear blood flow: measurements techniques. Am J Otolaryngol 9:291-301

28. Nuttall AL, Lawrence M (1980) Endocochlear potential and scala media oxygen tension during partial anoxia. Am J Otol $1: 147-153$

29. OhIsén KA, Baldwin DL, Nuttall AL, Miller JM (1991) Influence of topically applied adrenergic agents on cochlear blood flow. Circ Res 69:509-518

30. OhIsén KA, Didier A, Baldwin D, Miller JM, Nuttall AL, Hultcrantz E (1992) Cochlear blood flow in response to dilating agents. Hear Res 58:19-25

31. Ohlsén A, Hultcrantz E, Engstrom B (1993) The effect of topical application of vasodilating agents on cochlear electrophysiology. Acta Otolaryngol (Stockh) 113:55-61

32. Puel JL, Bobbin RP, Fallon M (1990) Salicylate, mefemanate, meclofemanate and quinine on cochlear potentials. Otolaryngol Head Neck Surg 102:66-73

33. Quirk WS, Avinash G, Nuttall AL, Miller JM (1992) The influence of loud sound on red blood cell velocity and blood vessel diameter in the cochlea. Hear Res 63:102-107

34. Ren TY, Laurikainen EA, Quirk WS, Miller JM, Nuttall AL (1993) Effect of electrical simulation of the superior cervical ganglion on cochlear blood flow in guinea pig. Acta Otolaryngol (Stockh) 113:146-151

35. Reuse-Blom S, Drieux K (1986) Effet de l'extrait de ginkgo biloba sur le spasme artériolaire chez le lapin. Presse Med 15: $1520-1523$

36. Shehata WE, Brownell WE, Dieler R (1991) Effects of salicylate on shape, electromotility and membrane characteristics of isolated outer hair cells from guinea-pig cochlea. Acta Otolaryngol (Stockh) 111:707-718

37. Shone G, Altschuler RA, Miller JM, Nuttall AL (1991) The effect of noise exposure on the aging ear. Hear Res 56:173-178 
38. Spoendlin H (1981) Autonomic innervation of the inner ear. Adv Otorhinolarynngol 27:1-13

39. Stypulkowski PH (1990) Mechanisms of salicylate ototoxicity. Hear Res 46:113-146

40. Thorne PR, Nuttall AL (1987) Laser Doppler measurements of cochlear blood flow during loud sound exposure in the guineapig. Hear Res 27:1-10
41. Thorne PR, Nuttall AL (1989) Alterations in oxygenation of cochlear endolymph during loud sound exposure. Acta Otolaryngol (Stockh) 107:71-79

42. Vane JR (1971) Inhibition of prostaglandins synthesis as a mechanism of action for aspirin-like drugs. Nature Rev Biol 231:232235 